\title{
Ulrich Brand
}

\section{Kapitalistisches Wachstum und soziale Herrschaft Motive, Argumente und Schwächen aktueller Wachstumskritik ${ }^{1}$}

Die multiple Krise fördert seit einigen Jahren Debatten um den Zusammenhang von wirtschaftlichem Wachstum, ausgedrückt in der jährlichen Zunahme des Bruttoinlandsprodukts, und steigendem Wohlstand. Eine enge Korrelation, so die übereinstimmende Annahme, werde problematisch.

So intensivierten sich etwa politiknahe wissenschaftliche Diskussionen um bestehende und gegebenenfalls zu ändernde Formen der Wohlstandmessung. Der Entwicklung geeigneter Indikatoren über die enge Bindung von Wirtschaftswachstum und Wohlstand hinaus liegt die normative Annahme zugrunde, dass „(d)eveloping better measures (d.h. Indikatorensysteme, U. B.) is not an end in itself but a means to enhance policies that improve people's lives." (OECD 2011: 4; vgl. auch EU-Kommission 2009) Der Deutsche Bundestag hat Anfang 2011 eine Enquete-Kommission eingerichtet, unter anderem zur „Entwicklung eines ganzheitlichen Wohlstands- bzw. Fortschrittsindikators" (Einsetzungsbeschluss, Drucksache 17/3853 des Deutschen Bundestags: 3). Dabei geht es im Kern nicht um Messung (bzw. das sollte nicht der Kern sein, auch wenn viele ÖkonomInnen sich darauf konzentrieren), sondern um andere Formen des individuellen und gesellschaftlichen Wohlstands. Es wird davon ausgegangen, dass Wohlstand eben nicht nur an der in Preisen ausgedrückten gesellschaftlichen Wirtschaftsleistung und individuellem Einkommen, die wiederum auf zunehmender Erwerbsarbeit und Arbeitsproduktivität basieren, hängt. Andere Dimensionen sind Gesundheit und Bildung, Lebenserwartung bzw. die Länge eines gesunden Lebens, intakte soziale Kontakte, die Qualität der Arbeit sowie ein nicht schädigendes Verhältnis zwischen Arbeit und Nicht-Arbeit (work-life-balance), Chancengerechtigkeit, persönliche Sicherheit und individuelle Freiheiten, politische Partizipationsmög-

1 Alex Demirović und Andrei Draghici aus der PROKLA-Redaktion und den Teilnehmenden des von Sabine Pongratz und Markus Wissen organisierten Jour Fixe zu Politischer Ökologie an der Hochschule für Wirtschaft und Recht danke ich für hilfreiche Anmerkungen. 
lichkeiten und lebenswerte Umwelt (OECD 2011, SSFC 2009, EU-Kommission 2009). Ebenfalls auf der politischen Ebene sind Initiativen wie „Beyond GDP“ der EU-Kommission (www.beyond-gdp.eu) oder "Wachstum im Wandel“ der österreichischen Regierung (www.wachstumimwandel.at) wichtige Beiträge zur Diskussion um Wirtschaftswachstum.

Die Frage, welches Wachstum und welcher Wohlstand gesellschaftlich gewünscht ist, wird also intensiv diskutiert - und sie ist keineswegs neu (vgl. Reuter 2012, Lötzer/Reuter 2012, Adler/Schachtschneider 2012, Steurer 2010, Schmelzer/Passadakis 2011, APuZ 2012). ${ }^{2}$ Wir verfügen zudem bereits über ein breites Set an Indikatoren hinsichtlich der Fragen sozialer Ungleichheit, Qualität der Arbeit oder (Nicht-)Nachhaltigkeit. Dennoch findet die Diskussion in einem veränderten Kontext statt, nämlich in der multiplen Krise des neoliberalen Kapitalismus. Damit öffnet sich der Raum für ein umfassend anderes gesellschaftliches Wohlstandverständnis und der damit einhergehenden Transformation der Produktions- und Lebensweise. Und in gewisser Weise konturieren sich in der Debatte um Wohlstand und den Stellenwert von Wirtschaftswachstum zu dessen Erreichung wichtige Dissense innerhalb des politischen und wirtschaftlichen Establishments (vgl. Miegel 2010, APuZ 2012, Konsens- und Sondervoten in Enquete-Kommission 2013).

Die weitreichendsten Beiträge sind im Umfeld einer Debatte angesiedelt, die unter dem Begriff Postwachstum, décroissance bzw. degrowth geführt wird (Martínez Alier et al. 2010 zur Geschichte der Diskussion). Die PostwachstumsPositionen kritisieren die aktuell dominierenden wirtschaftspolitische Antworten auf die Krise - sei es qua neoklassischer Austeritäts- oder keynesianischer Nachfrage- und Umverteilungspolitik: Dass nämlich der „Wachstumsmotor“ wieder anlaufen und entsprechend die ökonomische Wettbewerbsfähigkeit erhöht werden müsse. Die Postwachstums-Debatte hält dem entgegen: Wachstum wird in Zeiten der multiplen Krise und insbesondere unter Bedingungen des Finanzmarktkapitalismus selbst zum destabilisierenden Faktor. Zudem erhöht sich die intra- und intergenerationelle Ungerechtigkeit, etwa durch zunehmenden Statuskonsum und positionalen Wettbewerb heute oder - auf die Zukunft bezogen - die Verschlechterung der Lebensbedingungen künftig lebender Menschen (Muraca 2012). Aber eben auch das weitere Wachsen der Produktion von Gütern und Dienstleistung, insbesondere von kurzlebigen Gütern, schafft potenzielle und reale Instabilität. Die Ressourcen müssen beschafft werden, was immer weniger

2 Ich beziehe mich in diesem Beitrag nicht auf konservative Diagnosen wie prominent jene von Meinhard Miegel. Der sieht die Ursachen des Probleme generierenden Wirtschaftswachstums allgemein im Schuldenmachen und dem „Wunsch nach schnellen Geld“ (2010: 15) und einen wesentlichen Treiber im überbordenden Konsum. 
konfliktfrei und ausschließlich über den Markt abläuft; der Klimawandel schafft viele Unsicherheiten inklusive der berüchtigten „Kipppunkte“ des lokalen oder regionalen Klimas.

Als Konsens der Debatte könnte gefasst werden: Degrowth ist „a multi-faceted political project that aspires to mobilise support for a change of direction, at the macro-level of economic and political institutions and at the micro level of personal values and aspirations. Income and material comfort is to be reduced for many along the way, but the goal is that this is not experienced as welfare loss.“ (Kallis, 2011: 878) Normative Prinzipien wir Kooperation und soziale Gerechtigkeit werden als zentral erachtet. Es geht um einen „voluntary, smooth and equitable transition to a regime of lower production and consumption." (Schneider et al., 2010: 511; Kallis, 2011: 574) Das ist ein zentraler Aspekt, den es den KritikerInnen entgegenzuhalten gilt: Postwachstum als Horizont bedeutet nicht, sich an Krisen zu laben und über den Niedergang industrieller Produktion politisch zufrieden zu sein. Postwachstum ist ein gesteuerter Prozess hin zu einer anderen, sozial-ökologisch nachhaltigen, gerechten und solidarischen Produktions- und Lebensweise. Die Anfang September in Leipzig stattfindende Vierte Internationale Degrowth-Konferenz für ökologische Nach haltigkeit und soziale Gerechtigkeit wird diesbezüglich ein wichtiger wissenschaftlicher und politischer Kristallisationspunkt werden (leipzig.degrowth.org/de/).

In diesem Beitrag umreiße ich knapp die Motive der sich entwickelnden Wachstumskritik und kritisiere einen Schwachpunkt des Großteils der Debatte: Herrschaftstheoretische und -kritische Perspektiven sind schwach oder allenfalls indirekt vorhanden. Das hat politische Implikationen, auf die ich am Ende eingehe. Dabei greife ich auf feministische und historisch-materialistische Einsichten zurück. Es geht mir weniger um Differenzen innerhalb feministischer und marxistischer Beiträge und zwischen ihnen, sondern darum, den analytischen Mehrwert dieser Perspektiven für die Postwachstums-Debatte hervorzuheben. An einer Stelle gehe ich auf eine wichtige, aber meines Erachtens im Kern verfehlte linke Kritik der Wachstumskritik ein.

\section{Motive der Wachstumskritik}

Unter Wachstumskritik fasse ich jene Vorschläge, die aus unterschiedlichen Gründen die Orientierung an ökonomischem Wachstum und die damit verbundenen Implikationen für problematisch halten. Zugespitzt könnte man diese Beiträge so zusammenfassen, dass Wirtschaftswachstum und die damit verbundenen - höchst unterschiedlich eingeschätzten - gesellschaftlichen Triebkräfte zu ökologischen, sozio-ökonomischen, politischen, friedenspolitischen, aber auch individuellen 
(psychischen wie auch die Lebensführung betreffenden) Risiken und Momenten wirtschaftlicher und gesellschaftlicher De-Stabilisierung werden. Die ökologische Krise, die aktuelle Wirtschafts- und Finanzkrise, der wachsende Druck auf die Menschen im Arbeitsleben, aber auch zur Prestigegewinnung, die gesellschaftliche Polarisierung, die ökologische Krise, zunehmende Gewalt bei Konflikten oder gar Kriegen um Ressourcensind dafür angeführte Belege. Das betrifft auch zeitliche Verschiebungen möglicherweise erst in Zukunft eintretende Probleme und Krisen.

Die Motive und Argumente einer grundlegenden Kritik am Wirtschaftswachstum als geeignete wirtschaftspolitische Bezugsgröße sowie als Indikator für Wohlstand und Lebensqualität sind vielfältig (vgl. mit etwas anderer Systematik Pennekamp 2011). Am bedeutendsten ist der seit etwa 40 Jahre bestehende und sich ausdifferenzierende Diskussionsstrang der ökologischen Grenzen des Wachstums (womit zunächst nicht das Wirtschaftswachstum, sondern der steigende Ressourcenverbrauch gemeint war). Ausgehend von der Studie „Grenzen des Wachstums" (Meadows et al. 1972) entwickelte sich eine intensive wissenschaftliche und gesellschaftspolitische Debatte über die zerstörerischen Implikationen auf zunehmender Ressourcennutzung basierender wirtschaftlichen Aktivitäten. Es gibt, so die zentrale Annahme dieser Beiträge, Knappheiten und Grenzen von Ressourcen und Möglichkeiten der Energieumwandlung. Diese Diskussion wird seit den 1990er Jahren ergänzt durch die Grenzen der Aufnahmefähigkeit von Emissionen in der Atmosphäre und Stratosphäre bzw. die Re-Absorption durch die sog. ,Senken“, insbesondere Wälder und Meere - prominent wird hier der Klimawandel angeführt. Seit den 2000er Jahren wird diese Perspektive ergänzt durch die als problematisch eingeschätzte Zerstörung zusammenhängender Ökosysteme (vgl. zu den unterschiedlichen Dimensionen die prominente Studie von Rockström 2009 zu den „planetarischen Grenzen“).

Hier setzt die neuere ökologisch ausgerichtete Post-Wachstumsdebatte an. Prominent ist etwa das Buch „Wohlstand ohne Wachstum“ von Tim Jackson (2011), in dem er fragt, wie in einer Welt mit weiterhin hohem Bevölkerungswachstum und begrenzten Ressourcen Wohlstand beschaffen sein soll und kann, ein „Wohlstand, der es den Menschen ermöglicht, ein gutes Leben zu führen, mehr Zusammenhalt in der Gesellschaft zu schaffen, mehr Wohlbefinden zu erfahren und trotzdem die materiellen Umweltbelastungen zu reduzieren." (ebd.: 54) Dabei vertraut er weder auf wirtschaftliches Wachstum, noch auf technologische Lösungen, denn sie seien eine Selbsttäuschung. Ein nachhaltiges Wirtschaftssystem bedarf politischer Rahmenbedingungen wie etwa einer ökologischen Steuerreform und strikte Obergrenzen für den Verbrauch von Ressourcen und den Ausstoß von Emissionen, kulturelle Veränderungen wie etwa den Abbau des Konsumismus, Verkürzung der Erwerbsarbeitszeit, den Abbau von Ungleichheit, die Stärkung der Fähigkeiten und des Sozialkapitals der Menschen sowie Un- 
terstützung der südlichen Länder beim Umbau ihrer Ökonomien (ebd.: 175ff.; ähnlich Martínez Alier et al. 2010, Muraca 2013).

Einen zweiten Strang der jüngeren Diskussion bildet die so genannte Glücksforschung, die sich in den letzten Jahren rasch entwickelt hat und die instruktive These vertritt, dass ab einem bestimmten Punkt die Höhe des Einkommens kaum mehr mit wachsender Lebenszufriedenheit korreliert. Die GesundheitsforscherInnen Richard Wilkinson und Kate Pickett (2010) zeigen mit dichtem empirischem Material: „Wirtschaftswachstum war für lange Zeit Motor des Fortschritts, doch in den reichen Ländern ist dieser Antrieb inzwischen weitgehend erschöpft. Das ökonomische Wachstum ist nicht mehr wie einst von Maßnahmen für das Wohlergehen und Wohlbefinden der Bürger begleitet. Schlimmer noch: Langfristig haben Ängste, Depressionen und andere soziale Probleme mit wachsendem Wohlstand zugenommen." (ebd.: 20; ähnlich SSFC 2009) Mehr Gleichheit, so ihre grundlegende These des internationalen Vergleichs, schafft bessere soziale Beziehungen, so wie umgekehrt gesundheitliche und soziale Probleme in Ländern mit großen Einkommensdifferenzen signifikant stärker sind. „Gesundheitliche und soziale Probleme treten am häufigsten in den ärmeren Schichten jeder Gesellschaft auf, darüber hinaus sind aber Gesellschaften mit signifikant hoher Ungleichheit von den genannten Problemen stärker betroffen." (ebd.: 35, 61) Wirtschaftswachstum verschärft demgegenüber soziale Probleme, da er in Gesellschaften mit weitgehend gesicherten Grundbedürfnissen zur Entstehung oder Entfaltung von Druck, Konkurrenz und Konsumismus beiträgt. Entsprechend benötigen die Menschen einen anderen Blick auf ihre eigenen Gesellschaften, um überhaupt ein breiteres Verständnis von Lebensqualität zu erhalten.

Ähnlich argumentieren die ÖkonomInnen Bruno S. Frey und Claudia Frey Marti (2010). Sie gehen davon aus, dass jede Person eigenständig definiert, was Glück im Sinne von Wohlbefinden oder Lebenszufriedenheit für sie bedeutet und welche gesellschaftlichen Rahmenbedingungen dafür notwendig sind. Die entsprechende Forschung möchte dazu beitragen, „Voraussetzungen zu schaffen, dass der Einzelne sein ganz persönliches Glück finden kann.“ (ebd.: 22, vgl. auch Layard 2009) Zeitliche und kulturelle Differenzen werden berücksichtigt. Dimensionen des Glücks sind neben persönlicher Veranlagung sozio-demographische Faktoren wie das Alter, wirtschaftliche wie das Einkommen, politische Einflüsse sowie kontextuelle und kulturelle bzw. religiöse Dimensionen. Am bedeutsamsten sind die Faktoren Arbeitsmarkt, materieller Lebensstandard, Freunde und Familie, Freizeit sowie Gesundheit. ${ }^{3}$ Auch die OECD konstatiert (2011: 265), dass "people are the best judges of how their own lives are going."

3 Empirisch gemessen wird das Glück etwa über Umfragen als „subjektives Wohlbefinden“ oder über objektivierbare Gefühlsäußerungen via Gehirnströme. Die subjektive 
Ein dritter Strang, der an die beiden ersten anschließt, könnte mit dem Buch von Niko Paech überschrieben werden: „Befreiung vom Überfluss“ (2012, vgl. auch Paech 2005). Motive der ökologischen Kritik und der Glückforschung werden aufgenommen und in eine radikale Diagnose sowie eine attraktive Botschaft übersetzt. Die Diagnose lautet, dass die Menschen in den westlichen Gesellschaften über ihre Verhältnisse leben hinsichtlich der eigenen Leistungsfähigkeit und der lokal und regional vorhandenen Ressourcen. Das räumlich ausgreifende Wirtschaftssystem geht einher mit Investitionen, entsprechenden Krediten und zu bedienenden Zinsen. Deshalb muss die Wirtschaft wachsen. Die Botschaft lautet: Die notwendige Reduktion des Ressourcenverbrauchs und der Nutzung der Senken durch weniger und andere industrielle Produktion kann mit einer stabileren Versorgung und mehr Glück im Sinne von subjektivem Wohlbefinden einhergehen. Das bedarf kultureller Veränderungen, insbesondere einer „kreativen Subsistenz" durch Eigenproduktion, Gemeinschaftsnutzung und längerer Nutzungsdauer (Paech 2012: 120ff.). Auf der Seite der Unternehmen kann der Wachstumszwang gemildert werden, indem es zu einer geringeren Spezialisierung kommt, da dann die Produktion weniger kapitalintensiv ist und entsprechend weniger Kredite getilgt und verzinst werden müssen. Zudem gehe es um lokale und regionale Produktion, d.h. um kürzere und entflochtene Produktionsketten, damit Nähe und Vertrauen entstehen, „welches per se eine weniger zins- und renditeträchtige Kapitalbeschaffung ermöglicht." (ebd.: 108) Dazu kommen etwa die Reduktion und Umverteilung der Arbeitszeit und entsprechend dem veränderten Konsumverhalten langlebige Konsumgüter.

Weitere Beiträge setzen sich spezifisch mit der Wirkung des Geldes sowie der Wirtschafts- und Finanzmarktkrise auseinander (etwa Binswanger 2009, NEF 2010). Hier wird argumentiert, dass die in der Geldwirtschaft strukturell verankerten Ansprüche auf Verzinsung zum Zwang zu Wirtschaftswachstum und tendenziell zu Instabilität führen.

Ein Diskussionsstrang spielt in vielen Beiträgen eine Rolle, steht aber in der sozialpsychologischen Zuspitzung für sich und erfährt derzeit einige gesellschaftliche Resonanz: Ursache aktueller Probleme und Ansatzpunkte für Veränderungen sind die Individuen. Prominent steht hier aktuell die Position von Harald Welzer. Er weist mit dem Begriff der „mentalen Infrastruktur“ (Welzer 2011) darauf hin, dass Konsum- und Wachstumsorientierungen tief verankert sind und mithin gesellschaftliche Veränderungen auch sozial-psychologische, habituelle und kulturelle Dimensionen haben. Konsum bedeutet Status und Sinnstiftung. Politische Steuerung kommt entsprechend rasch an Grenzen. Der Vorschlag von

Lebenszufriedenheit wird auch in Indizes wie dem World Values Survey, dem Gallup World Poll oder dem Happy Planet Index der New Economics Foundation dargestellt. 
Welzer und dem Projekt von „Futur Zwei“ lautet, dass es einer neuen Erzählung (Story) bedarf, also ein emotionales und identitätsträchtiges Angebot um die Frage, wie im Jahr 2025 gelebt werden soll (ebd.: 40).

\section{Zwischenfazit}

Konsens in der wachstumskritischen Diskussion ist, dass es der gesellschaftlichen Veränderungen bedarf, um vielfältige soziale und ökologische Probleme zu bearbeiten. Dabei auf Wachstum zu setzen, ist wohl eher Teil des Problems als der Lösung. Der Durchgang durch wichtige Beiträge der vor allem deutschsprachigen oder ins Deutsche übersetzte internationale Wachstumskritik zeigt, dass es eine facettenreiche Wohlstandsdiskussion gibt, für die Wohlstand ohne Wachstum vorstellbar, umsetzbar und unabdingbar ist. ${ }^{4}$

Die unterschiedlichen Beiträge sind zudem ein Angriff auf die MainstreamWirtschaftswissenschaft, da sie zum einen den nutzenmaximierenden homo oeconomicus, zum anderen die Zentralität des Wirtschaftswachstums hinterfragt. Und zudem wird kritisiert, dass der Fokus auf die Produktion und den geldvermittelten Austausch von Gütern und Dienstleistungen viel zu eng ist.

Allerdings läuft die wachstumskritische Debatte Gefahr, aus meiner Sicht zentrale Momente wirtschaftlichen, d.h. kapitalistischen Wachstums zu unterschätzen; nämlich ihren herrschaftlichen Gehalt. Das deutet sich in vielen Beiträgen mit Begriffen wie „Befreiung von Herrschaft“, „Gleich heit ist Glück “ oder den tief verankerten „mentalen Infrastrukturen“ an. Doch es bedarf m.E. einer weiteren Präzisierung, was unter kapitalistischen Wachstum bzw. Kapitalismus überhaupt verstanden wird. Viele Beiträge bleiben hier unbestimmt und verstehen unter Wachstum die Zunahme von materieller Produktion und Konsum, ohne nach deren Charakter als kapitalistische, patriarchale, rassifizierte oder postkoloniale soziale Verhältnisse zu fragen. Beispielsweise fragt sich Tim Jackson (2011: 200) ganz am Ende seines prominenten Buches, was unter Kapitalismus verstanden werden sollte und stellt fest: „Das ist gar nicht so einfach.“

Deutlich wird diese Auslassung beispielsweise am breit rezipierten und in vielen Punkten plausiblen Ansatz von Niko Paech, der davon ausgeht, dass die Menschen in den materiell wohlhabenden Gesellschaften unter dem Überfluss eher leiden und dadurch die ökologischen Grenzen erreicht werden. „Befreiung vom Überfluss" lautet die Option und Aufforderung. Es wird durchaus die mit

4 Die internationale Diskussion wird neben Büchern vor allem in der Zeitschrift Ecological Economics geführt; vgl. auch die Sonderausgaben von Futures August 2012, Journal of Cleaner Production Frühjahr 2013 und Environmental Values April 2013 und einige Beiträge in Monthly Review. 
dem gegenwärtigen Wirtschafts- und Wachstumsmodell einhergehende Macht der Großunternehmen gesehen (und entsprechend auf eine Regionalisierung der Wirtschaft gesetzt). Doch in der Diagnose fehlt weitgehend, inwieweit sozioökonomische, kulturelle und politische Herrschaft sowie die in den Naturverhältnissen angelegte Herrschaft zur Stabilisierung gegenwärtiger Verhältnisse beitragen - samt ihrer sozialstrukturellen Implikationen - und welche Konsequenzen das hätte.

Mein Vorschlag lautet, dass die wachstumskritische Debatte fruchtbarer geführt werden kann, wenn Wachstum in Verbindung mit der herrschenden kapitalistischen Produktions- und Lebensweise gesehen wird. Diese ist nicht nur ein Produktions- und Konsumsystem, sie ist auch ein System von Macht und Herrschaft - auch und gerade über die Natur. Eigenartigerweise wird dieser Aspekt kaum thematisiert. Geschieht dies aber, kann auch die in jüngster Zeit beginnende Diskussion um Wachstum und Demokratie (etwa Muraca 2012, Asara et al. 2013) gehaltvoller geführt werden.

\section{Wachstum und Herrschaft}

Damit sind wir an einem Punkt, der in der Debatte um Wachstum, Wachstumskritik und Alternativen bislang kaum eine Rolle spielt: Nämlich der Zusammenhang von wirtschaftlichem Wachstum und kapitalistischer Form von Akkumulation und Herrschaft. Die mannigfaltigen sozialen Verhältnisse, die eine Orientierung am Wirtschaftswachstum erfordern und sich dadurch gleichzeitig absichern, sind der kapitalistische Markt verstanden als soziales Verhältnis und damit verbunden Klassen-, rassifizierte und Geschlechterverhältnisse und postkolonial aufgeladene globale Zusammenhänge. Auch eine bestimmte Form der Politik, nämlich eine an Staat und Parteien ausgerichtete, welche sich stark an der Schaffung politisch angemessener kapitalistischer Wachstumskonstellationen orientiert, ist Herrschaft.

Feministische und marxistische Beiträge betonen - in der hier jeweils vorgenommenen Lesart - eben diesen herrschaftlichen Charakter von Wachstum. Wirtschaftswachstum und die Orientierung daran sind Ausdruck tief verankerter sozialer Verhältnisse.

Eine Unterscheidung ist hier wichtig: Dem Kapital geht es nicht zuvorderst um Wachstum, sondern um Profit. Die in der Wachstumsdebatte implizit thematisierte Dynamik der Mehrwertproduktion und Kapitalakkumulation - wie auch die Krisenhaftigkeit von Gesellschaften unter Dominanz der kapitalistischen Produktionsweise bestehen zuvorderst darin, dass sich historisch eine Klasse von Eigentümern an Produktionsmitteln und anderen Vermögen herausgebildet hat, 
die daran interessiert ist, aus ihrem Geld und Kapital mehr Geld und Kapital zu machen. Es ist also nicht nur der Kredit und dessen Verzinsung und Tilgung problematisch, wie in vielen wachstumskritischen Beiträgen argumentiert wird, sondern die damit einhergehenden sozialen Produktions-, Eigentums- und Lebensverhältnisse.

Die überwiegende Mehrheit der Menschen besitzt keine Produktionsmittel, Ersparnisse, die vom Zwang zur Lohnarbeit befreien, oder Vermögen, sondern reproduziert sich durch Lohnarbeit, die die kapitalistischen Waren produziert. In Gesellschaften mit kapitalistischer Produktionsweise basiert die Reproduktion der Lohnarbeit und der Menschen selbst in ihren unterschiedlichen Lebensphasen quer durch alle Schichten nicht nur auf dem Lohn, sondern auch auf der oft von Frauen geleisteten Haus- und Pflegearbeit. Dies wiederum hängt an asymmetrischen Geschlechterverhältnissen, an gesellschaftlichen Bewertungen von „wertvoller“ und „nicht-wertvoller“ Arbeit (vgl. etwa Jochimsen/Knobloch 2006). Das Thema (Erwerbs-)Arbeit bildet aber bislang keinen zentralen Bezugspunkt der Postwachstums-Diskussion (zur Bedeutung der Postwachstumsdebatte für die Arbeitssoziologie insgesamt vgl. Mahnkopf 2012).

Markt und Ökonomie konstituieren entsprechend Herrschaftsverhältnisse entlang von Klassen-, Geschlechter- und ethnischen Linien, der Markt ist ein „bestimmtes gesellschaftliches Kräfteverhältnis in einer bestimmten Struktur des Produktionsapparates"(Gramsci GH 8: 1015, GH 10: 1281). Hegel (1807/1986: 143-146) hat in seiner knappen Analyse von Herrschaft und Knechtschaft das Argument der gegenseitigen, aber asymmetrischen Anerkennung und Abhängigkeit von Herrschenden und Beherrschten entwickelt. Der Herrschende erreicht seine Freiheit durch die Arbeit des Beherrschten, die wiederum zu gesellschaftlichem Fortschritt führt. Diesen Gedanken hat der junge Marx für die aufkommende Klassengesellschaft übernommen. Die meisten Menschen erkennen, weitgehend unfreiwillig und machtlos, als Lohnabhängige nicht nur die kapitalistische Wachstumsmaschinerie an, sondern eben die darunter liegenden Herrschafts- und Eigentumsverhältnisse. Er spitzte das folgendermaßen zu: „Die soziale Macht, d.h. die vervielfachte Produktionskraft, die durch das in der Teilung der Arbeit bedingte Zusammenwirken der verschiedenen Individuen entsteht, erscheint diesen Individuen, weil das Zusammenwirken selbst nicht freiwillig, sondern naturwüchsig ist, nicht als ihre eigne, vereinte Macht, sondern als eine fremde, außer ihnen stehende Gewalt, von der sie nicht wissen woher und wohin, die sie also nicht mehr beherrschen können, die im Gegenteil nun eine eigentümliche, vom Wollen und Laufen der Menschen unabhängige, ja dies Wollen und Laufen erst dirigierende Reihenfolge von Phasen und Entwicklungsstufen durchläuft“(MEW 3: 34).

Gramsci hat vor diesem Hintergrund seine Hegemonietheorie ausgearbeitet. Die materiellen wie ideologischen Konsenselemente von Herrschaft werden durch 
den „Alltagsverstand“ (Gramsci GH 3: 372) stabilisiert, der eben zentrale Dimensionen sozialer Herrschaft als nicht hinterfragbar erscheinen lässt. Hegemonie in diesem Sinne ist eine umfassende materielle Praxis, „nämlich die täglichen Initiativen vieler einzelner und sozialer Gruppen, in denen sie in der Form einer aktiven Selbstunterwerfung unter die gemeinsam geteilten Gewohnheiten großer Kollektive die Zustimmung zu Herrschaft erkennen lassen“(Demirović 1997: 257).

Kapitalistische Dynamik besteht zudem darin, immer weitere Aspekte der Gesellschaft in marktfähige Waren zu verwandeln und darüber Macht und Herrschaft zu konstituieren. Das betrifft neben der Natur gerade die Menschen, die ihre Arbeitskraft verkaufen müssen in kapitalistischen Unternehmen. In China und Indien wird das als Wirtschaftswunder gefeiert, ohne eben nach dem sozialen Gehalt und der enormen Wucht zu fragen, die für die Menschen „eine fremde, außer ihnen stehende Gewalt" ist. Doch auch dieser Aspekt, die Kommodifizierung von Arbeitskraft und Natur, wird in der Postwachstums-Debatte nicht systematisch behandelt.

Zudem gilt es aus hegemonietheoretischer Perspektive zu berücksichtigen, dass die sozialen Herrschaftsverhältnisse im Bewusstsein der dieser Herrschaft Unterworfenen nicht als solche wahrgenommen werden, sondern als stummer Zwang anonymer Verhältnisse, als kaum zu steuernde Prozesse von technischem Fortschritt und globalem Markt, von Produktivismus und Globalisierung. Die meisten Menschen erleben ihren Alltag als wenig handlungsfähige Individuen; Handlungsantriebe sind neben der Einkommenssicherung am ehesten solche der Statussicherung (Welzer 2011), die wiederum die imperiale Lebensweise (Brand/ Wissen 2011a) absichern. Das ist die Basis kapitalistischer Kultur.

Schließlich ist das kapitalistische Wirtschafts- und Gesellschaftssystem systematisch blind gegenüber den Voraussetzungen und Folgen des Wirtschaftens. In der feministischen Diskussion wird davon ausgegangen, dass die kapitalistische Ökonomie eine Ökonomie der Trennung ist, weil die formellen Marktprozesse von ihren Voraussetzungen - nämlich der nicht-bezahlten Arbeit, insbesondere der Sorgearbeit, und den Elementen der Natur, die keine Waren sind - getrennt werden (Biesecker et al. 2012). Insofern ist Externalisierung ein „Prinzip“, das entscheidend zum Funktionieren der kapitalistischen Wirtschaft beiträgt. Wirtschaftswachstum ist zudem eng verbunden mit einem männlichen, rationalistischen und westlichen Entwicklungsverständnis, das zuvorderst und als Bestandteil patriarchaler Dominanzverhältnisse an der Beherrschung der Natur orientiert ist.

Kurz: Eine systematischere herrschaftstheoretische Diskussion, die hier nur angedeutet ist und um subjekt-, hegemonie- und staatstheoretische Dimensionen ergänzt werden müsste, könnte der Postwachstumsdebatte eine umfassendere und kritischere Ausrichtung geben. Einhergehen müssten entsprechende Un- 
tersuchungen auch mit der Frage, welche Rolle das Militär bzw. der militärischindustrielle Komplex in der Aufrechterhaltung des Wachstumsparadigmas hat.

\section{Ökologische Krise und Naturbeherrschung}

Ein Großteil der Beiträge zu Postwachstum verortet sich im wissenschaftlichen Paradigma der Ökologischen Ökonomie. 5 Sie beziehen sich auf deren Gründerfiguren wie Robert Constanza (1991) und Herman Daly (1996; vgl. etwa das Lehrbuch von Commons/Stagl 2005, Überblick und Kritik bei Spash 2012). Das hat weitreichende Implikationen. Denn die Ökologische Ökonomie - das berühmte Modell umfasst drei unterschiedlich große Kreise - nimmt an, dass die „Wirtschaft" (kleinster Kreis) eingebettet in der „Gesellschaft“ und diese beide eingebettet in der „bio-physikalischen Sphäre“ (größter Kreis) liegen.

Die ökologischen Begründungen für ein neues Wohlstandsverständnis gehen meist von einer Übernutzung bzw. Zerstörung der Natur, d.h. ihrer Ressource, ihrer ökosystemaren Dienstleistungen und ihrer Senken aus. Prominent steht hier der Begriff der „planetarischen Grenzen“ (Rockström et al. 2009), die bereits übernutzt werden oder bedroht sind. Die Menschheit und die einzelnen Gesellschaften sollen maximal so viel verbrauchen, dass das Ökosystem Erde sich ohne langfristigen Schaden alljährlich reproduzieren kann. Fragen sozialer Herrschaft und die praktische wie theoretische Kritik daran bleiben abgeschattet.

Die Politische Ökologie, die stark in einer feministischen und historischmaterialistischen Tradition steht, hat ein davon unterschiedenes Verständnis des Zusammenhangs von Wirtschaft, Gesellschaft und Natur und spricht - zumindest in der deutschsprachigen Diskussion - entsprechend von gesellschaftlichen Naturverhältnissen (Görg 2003, Dietz/Wissen 2009, Köhler/Wissen 2010, Brand/Wissen 2011b).

Der Stoffwechsel zwischen Gesellschaft, Individuum und Natur ist wechselseitig konstitutiv. In den Blick geraten damit nicht die „Umwelt“, sondern die herrschaftlich organisierte gesellschaftlichen Formen der Aneignung von Natur: also die Formen, in denen gesellschaftliche Basisbedürfnisse wie Ernährung und Wohnen, Mobilität und Kommunikation, Gesundheit und Fortpflanzung materiell und symbolisch produziert und konsumiert werden - und eben unter Bedingungen komplexer sozialer und damit herrschaftlicher Verhältnisse. Eine entscheidende Triebkraft der Naturaneignung - nicht die einzige! - ist der kapitalistische Akkumulationsimperativ. So tendiert die Ernährungsweise dazu,

5 Genauer müsste es heißen: Ökonomik, also Wirtschaftswissenschaft; im Englischen entspricht das den Ecological Economics. 
kommodifiziert und industrialisiert zu werden. Die Energieversorgung erfolgt eher - das können wir aktuell am roll-back der Energiewende in Deutschland besichtigen - unter Kontrolle mächtiger kapitalistischer Akteure statt. Politik findet eben nicht für „die Menschheit“ statt, sondern ganz konkret als Verfolgung von Interessen. Umweltfragen sind Macht- und Herrschaftsfragen.

Marx betonte, die Gebrauchswert schaffende Arbeit sei „ewige Naturnotwendigkeit, um den Stoffwechsel zwischen Mensch und Natur, also das menschliche Leben zu vermitteln“ (MEW 23: 57). Doch die gesellschaftliche Dynamik wird von der Produktion des Tauschwertes bestimmt. Die Ware, die den Tauschwert verkörpert, hat jedoch „mit ihrer physischen Natur und den daraus entspringenden dinglichen Beziehungen absolut nichts zu schaffen." (MEW 23: 86, auch 192ff.; vgl. Altvater 1991: 250, Dietz/Wissen 2009, Foster 2010) Ich komme auf diesen Punkt im anschließenden Exkurs nochmals zurück. Entsprechend wird das krisenhafte Verhältnis von Gesellschaft zur Natur anders gefasst als im Großteil der Ökologieund auch der Postwachstumsdebatte. Marx betonte, dass neben der Arbeitskraft auch die natürlichen "Springquellen alles Reichtums" untergraben werden (MEW 23: 530). Das berühmte Diktum der Dialektik der Aufklärung von Horkheimer und Adorno (1944/1989: 36) gilt bis heute: „Jeder Versuch, den Naturzwang zu brechen, indem Natur gebrochen wird (also sich aus den Abhängigkeiten von der Natur zu lösen, U.B.), gerät nur umso tiefer in den Naturzwang hinein (erhöht also die Abhängigkeit, U.B)." Das sehen wir heute: Der Versuch im Bereich erneuerbarer Energien, Erdöl durch „saubere“ Agrartreibstoffe zu ersetzen, führt zur Rodung von Regenwäldern oder zur Umwandlung kleinbäuerlich bewirtschafteter Landstriche in kapitalistisch reorganisierte Monokulturen. Die Grüne Ökonomie als Strategie und Versprechen ist der Versuch, eine neue Runde der Naturbeherrschung einzuläuten (vgl. Wichterich 2011, Brand 2009).

Die Postwachstums-Debatte sollte durch eine herrschaftstheoretische Fassung gesellschaftlicher Naturverhältnisse bereichert werden, um nicht die argumentative Figur zu reproduzieren, dass sich Gesellschaft und Natur gegenüberstehen und „die Gesellschaft“ mit ihren bestehenden sozio-ökonomischen und gesellschaftspolitischen Institutionen vermeintlich die „übernutzte Natur“ in den „planetarischen Grenzen“ halten sollte.

\section{Exkurs: Linke Kritik der Wachstumskritik}

Eine gewisse linke Kritik an wachstumskritischen Positionen trifft einerseits einen wichtigen Punkt, der in der Diskussion berücksichtigt werden sollte. Gleichwohl verfehlt sie aber den Kern der Postwachstumsdebatte.

Ulrich Busch (2013) weist in seiner Kritik an der Wachstumskritik zu Recht darauf hin, dass häufig die Zunahme des stofflichen Verbrauchs und des in Prei- 
sen ausgedrückten Tauschwerts verwechselt werden. Die ökologisch motivierte Wachstumskritik erliegt damit einem naturalistischen Kurzschluss, zumal sie Input-Größen (Ressourcen) mit Output-Größen (Wirtschaftsleistung) in eins setzt (ebd.: 127). Wirtschaftswachstum und damit zunehmende Wohlfahrt könne sehr wohl bei sinkendem Ressourcenverbrauch stattfinden. Daher empfiehlt er der Linken, sich mit Wachstumskritik zurückzuhalten. Theoretisch ist eine solche Entkopplung zwar möglich und wird von auf Technologie und „Effizienzrevolution" setzenden Positionen zwar immer wieder behauptet (knapper Überblick und Kritik im Kontext der Wachstumsdebatte bei Adler/Schachtschneider 2012). Allerdings ist seine Aussage falsch, dass sich bereits in der Vergangenheit das in Werten bzw. Preisen gemessene Wirtschaftswachstum vom Ressourcenverbrauch in vielen Bereichen absolut entkoppelt habe.

Die Erfahrungen lehren uns das Gegenteil. So wird etwa im Schlussbericht der erwähnten Enquete-Kommission des Bundestages (2013: 435-445 zu „systematischen Herausforderungen für Entkopplungsprozesse“) mit Bezug auf viele Studien gezeigt, dass die „Entkopplung“ von Wirtschaftswachstum und Ressourcenverbrauch bzw. Senkennutzung nur relativ, aber nicht absolut stattfindet. Eine umfassende Studie von UNEP (2011) bestätigt das. Zudem übergeht Busch bei seiner Feststellung, dass in der Bundesrepublik Deutschland seit 1978 das BIP preisbereinigt jährlich um 2 Prozent gewachsen, der Rohstoffverbrauch jedoch um 1,4 Prozent zurückgegangen sei, die intensive Forschung zu den „ökologischen Rucksäcken“, die messen, wie viel Ressourcenverbrauch in den importierten Vorprodukten enthalten ist (Dittrich 2010, Bringezu/Schütz 2010).

Das zentrale und aus meiner Sicht falsche Argument von Busch lautet: „Für die Zukunft ist bei vielen Ressourcen vom Diktum einer absoluten Senkung ihrer Inanspruchnahme auszugehen, ebenso wie bei der Emission von Schadstoffen und Abprodukten, wo die Belastungsgrenze erreicht ist." (Busch 2013: 130) Das ist jene Hofferei, wie sie von der Neoklassik wieder und wieder angeführt wird: Mit heute noch nicht bekannten Technologien soll jene Ressourcenproduktivität erreicht werden, mit der die ökologische Krise überwunden werde.

Die apodiktische und empirisch kaum belegte Aussage verfehlt zudem den Kern der Sache. Denn das Argument, dass eine linke Position nicht auf Wirtschaftswachstum verzichten sollte, geht an der Intention der Wachstumskritik vorbei. Es geht ja gerade nicht um ein Ja oder Nein zu Wirtschaftswachstum, sondern um einen genaueren Blick auf die Implikationen, Bedingungen und Konsequenten kapitalistischen Wachstums. 


\section{Ausblick}

Begriffe existieren nicht losgelöst von gesellschaftlicher Realität, von Interessen und Machtverhältnissen. Sie sind Ausdruck gesellschaftlicher Praxis und leiten diese an. Sie benennen, d.h. sie machen bestimmte Sachverhalte sichtbar; und sie entnennen, machen also andere unsichtbar. Das betrifft auf der gesellschaftlichen Ebene zentral den kaum hinterfragbaren Wachstumsbegriff. Bearbeitungen der multiplen Krise ohne Wirtschaftswachstum - das ist in öffentlichen und Fachdebatten fast undenkbar. Ähnliches gilt für den Fortschrittsbegriff (zur Ideengeschichte des Fortschritts vgl. Müller 2011, Zimmer 2011).

Dabei ließe sich an einen Gedanken Adornos anschließen: „Fortschritt heißt: aus dem Bann heraustreten, auch aus dem des Fortschritts, der selber Natur ist, indem die Menschheit ihrer eigenen Naturwüchsigkeit innewird und der Herrschaft Einhalt gebietet, die sie über Natur ausübt und durch welche die der Natur sich fortsetzt. Insofern ließe sich sagen, der Fortschritt ereigne sich dort, wo er endet." (Adorno 1977: 625) ${ }^{6}$ Das lässt sich umstandslos aufden Wachstumsbegriff übertragen. Auch dieser wirkt naturwüchsig.

Eine „wachstumsbefreite Gesellschaft“ (Wolfgang Sachs) hätte die unterschiedlichen Formen sozialer Herrschaft - klassen- und geschlechterspezifischer, rassistischer und internationaler - und ihrer Institutionalisierung wie etwa Markt, Staat, Familie anzugehen sowie die Herrschaft über Natur. Denn bislang ist die dominante Erfahrung der meisten Menschen, die Gesellschaft nicht gestalten zu können. An den Schalthebeln der politischen, ökonomischen und kulturellen Macht sitzen andere. Und die achten darauf, das ist in der aktuellen Krise zu berücksichtigen, dass das auch so bleibt. Angesichts dieser Konstellation bleiben Verweise auf einen stattfindenden Wertewandel hin zu nachhaltigen Konsummustern wichtig, aber eben auch etwas hilflos. Die Skizzen einer „solidarischen Postwachstumsökonomie“ bleiben meist herrschaftsvergessen.

Die auf Warentausch basierende Gesellschaft erzeugt die „rationale Gestalt der mythischen Immergleichheit“ (Adorno 1977: 636), verstellt Alternativen. Eine die verschiedenen herrschaftlichen Verhältnisse theoretische und praktische Wachstumskritik könnte einen Ausweg aus der , universalen Regression, die heute mit dem Fortschritt sich verbündet“ leisten (ebd.: 638).

Die Degrowth-Bewegung schafft aktuell in Ländern des globalen Nordens überhaupt wieder gesellschaftliche Vorstellungsräume, anderes als nur die Ausrichtung am Wirtschaftswachstum zu denken. Das ist ihr großes Verdienst.

6 Christoph Görg verwies auf diesen Gedanken in einer Diskussion zum Thema Wachstumskritik im Rahmen des in Fußnote 1 erwähnten Jour Fixe, in dem ich zentrale Gedanken dieses Textes skizzierte. 
Damit ist sie zentraler Bestandteil aktueller Strategiedebatten (vgl. etwa Biesecker et al. 2012, Klein 2013, Thie 2013). Aktuell entwickelt sich in Lateinamerika eine Art Komplement unter dem Begriff des „Post-Extraktivismus“(Lang/Mokrani 2013, Svampa 2013), der auch auf andere Weltregionen anwendbar ist. Beides verweist auf bestehende und zu schaffende Formen des Wohlstandbegriffs, der auf politische Gestaltung, sozial-ökologisch verträgliche Produktion und ein attraktives Leben für die Menschen setzt: Die de-stabilisierenden Formen des kapitalistischen Wachstums und die damit verbundenen Interessen müssen verändert werden. Damit werden gesellschaftliche Bedingungen möglich, unter denen Menschen ihre Individualität entfalten und leben können - und zwar in einem solidarischen sozialen Zusammenhang, der ja erst die Bedingung freier Persönlichkeitsentwicklung ist.

Die politisch progressive Position einer anderen, attraktiven Produktions- und Lebensweise hat sich durch die „fordistische Erfahrung“ eines auf Industrialismus, Fossilismus und imperialen Verhältnissen basierenden Wirtschafts- und Gesellschaftsmodells zu arbeiten. Sie kann bzw. sollte diese Erfahrung nicht mit Verachtung für „Konsumismus“ oder „Überfluss“ oder der Warnung vor dem „Verlassen der Komfortzone“ (Welzer 2013: 133, 237; wobei der Begriff suggeriert, dass dieses eh nicht geschehen wird) beiseite wischen, sondern ihre Widersprüchlichkeit sehen und zu politischen Anknüpfungspunkten machen. Die ambivalente liberale Demokratie war und ist eben auch eine „Carbon Democracy“ (Mitchell 2011), in der sich Machtverhältnisse entlang der Verfügung über fossile Energieträger strukturieren, aber in einigen Ländern eben auch Erfahrungen von Wohlstand für breite Bevölkerungsteile. Gerade deswegen scheint es zentral, auf der Frage der Demokratie im Sinne praktischer Herrschaftskritik und einer bewussten Gestaltung der Gesellschaft zu bestehen - insbesondere auf unterschiedliche Formen der Wirtschaftsdemokratie. Demokratische Prozesse hin zu Post-Wachstum bzw. die demokratische Transformation der Gesellschaft beinhalten attraktive und demokratisch gestaltete Formen von Ernährung und Kleidung, von Wohnen und Zusammenleben (programmatisch etwa Ihme 2010). Wie sehen nachhaltige Stadt-Land-Verhältnisse aus, was bedeutet solidarische Mobilität? Wie werden Nahrungsmittel nachhaltig, fair und weltweit in ausreichender Menge produziert und verteilt, die gut schmecken und gesund sind? Wie werden Konflikte ausgetragen mit den global players der transnationalen Nahrungsmittelindustrie, wenn Lebensmittel wieder lokal und regional produziert werden?

Demokratie bedeutet Gestaltung und Konflikt, Infragestellung vermeintlicher und Herrschaft sichernder Selbstverständlichkeiten, Veränderung und offene Zukunft, ,über die Gliederung des komplex gegliederten Ganzen der Gesellschaft selbst demokratisch zu entscheiden. Alle haben das gleiche Recht, die Koordi- 
nation des gesellschaftlichen Zusammenlebens festzulegen. Dies setzt einen demokratisch getragenen, umfassenden Transformationsprozess in Gang, der sich nicht auf Politik, die Gestalt des Volkssouveräns und den Nationalstaat beschränkt, sondern perspektivisch alle Bereiche des Zusammenlebens einbezieht." (Demirović 2013: 214; vgl. auch Brie 2014). Die als alternativlos postulierte Orientierung am Wirtschaftswachstum und die damit verbundenen Praktiken und Kräfteverhältnisse sind hier hinderlich. Deshalb sollten Herrschaftskritik und Fragen der Demokratie in der wachstumskritischen Debatte eine zentrale Rolle einnehmen.

\section{Literatur}

Adler, Frank; Schachtschneider, Ulrich (2012): Vom „nachhaltigen Wachstum“ zur „Postwachstumsgesellschaft"? In: Forum Wissenschaft 2.

Adorno, Theodor W. (1977): Fortschritt, in: ders.: Gesammelte Schriften. Band 10.2, Frankfurt/M., 617-638.

Altvater, Elmar (1991): Die Zukunft des Marktes. Ein Essay über die Regulation von Geld und Natur nach dem Scheitern des "real existierenden Sozialismus". Münster.

- (2011): Mit Green New Deal aus dem Wachstumsdilemma? In: Widerspruch 60, 119-132.

APuZ - Aus Politik und Zeitgeschichte (2012): Wohlstand ohne Wachstum? Heft Nr. 27; 28, Juli.

Asara, Viviana; Profumi, Emanuele; Kallis, Giorgos (2013): Degrowth, Democracy and Autonomy. In: Environmental Values (22(1), 217-240.

Biesecker, Adelheid; Wichterich, Christa; von Winterfeld, Uta (2012): Feministische Perspektiven zum Themenbereich Wachstum, Wohlstand, Lebensqualität. Materialie M-17(26)23 der Enquete-Kommission Wachstum, Wohlstand, Lebensqualität des Deutschen Bundestages.

Binswanger, Hans Christoph (2009): Vorwärts zur Mäßigung. Perspektiven einer nachhaltigen Wirtschaft. Hamburg.

Brand, Ulrich (2009): Schillernd und technokratisch. Grüner New Deal als magic bullet in der Krise des neoliberal-imperialen Kapitalismus? In: PROKLA 156, 475-481.

Brand, Ulrich; Wissen, Markus (2011a): Sozial-ökologische Krise und imperiale Lebensweise. In: Demirović, Alex et al. (Hrsg.): VielfachKrise im finanzdominierten Kapitalismus, Hamburg, 78-93.

- (2011b): Die Regulation der ökologischen Krise. In: Österreichische Zeitschrift für Soziologie 36(2), 12-34.

Brangsch, Lutz (2011): Kennziffernfragen sind Machtfragen. In: Kurswechsel 1, 25-38.

Brie, Michael (2014): Futuring. Transformation im Kapitalismus über ibn hinaus. Münster.

Bringezu, Stefan; Schütz, Helmut (2010): Der „ökologische Rucksack“ im globalen Handel. In: Geographische Rundschau 4, 12-17.

Busch, Ulrich (2013): Wachstum und Wohlfahrt, In: Berliner Debatte Initial 24(3), 122-137.

Common, Michael; Stagl, Sigrid (2005): Ecological Economics. An Introduction. Cambridge.

Demirović, Alex (1997): Demokratie und Herrschaft. Münster.

- (2013): Multiple Krise, autoritäre Demokratie und radikaldemokratische Erneuerung. In: PROKLA 171, 193-215.

Dietz, Kristina; Wissen, Markus (2009): Kapitalismus und „natürliche Grenzen“. Eine kritische Diskussion ökomarxistischer Zugänge zur ökologischen Krise. In: PROKLA 159, 351-370.

Dittrich, Monika (2010): Verlagert der Norden Umweltbelastungen in den Süden? in: Geographische Rundschau 4, 18-24. 
Enquete-Kommission (2013): Schlussbericht der Enquete-Kommission „Wachstum, Woblstand, Lebensqualität"des Deutschen Bundestages. Drucksache 13;300. Berlin: Deutscher Bundestag.

EU-Kommission (2009): Das BIP und mehr. Die Messung des Fortschritts in einer Welt im Wandel. $\operatorname{KOM}(2009) 433$.

Foster, John Bellamy (2010): Capitalism and Degrowth - An Impossibility Theorem. In: Monthly Review 62(8).

Frey, Bruno S.; Frey Marti, Claudia (2010): Glück. Die Sicht der Ökonomie. Zürich; Chur.

Görg, Christoph (2003): Regulation der Naturverhältnisse. Münster.

Gramsci, Antonio (191ff.): Gefängnishefte. Hamburg/Berlin.

Habermann, Friederike (2011): Ecommony statt Economy. In: informationen für diefrau, Oktober. Berlin: 17-19.

Hegel, Georg Wilhelm Friedrich (1807/1986): Phänomenologie des Geistes. Frankfurt/M.

Horkheimer, Max; Adorno, Theodor W. (1944/1989): Dialektik der Aufklärung. Frankfurt/M.

Ihme, Bernd (2010): Nachhaltige Entwicklung - sozial-ökologischer Umbau. Beiträge zur politischen Bildung 2/2010. Berlin: Rosa Luxemburg Stiftung.

ISM - Institut Solidarische Moderne (2011): Umrisse eines sozialökologischen Gesellschaftsumbaus auf dem Weg in eine Solidarische Moderne, www.solidarische-moderne.de

Jackson, Tim (2011): Wohlstand ohne Wachstum. Leben und Wirtschaften in einer endlichen Welt. München.

Kallis, Giorgos (2011): In defence of degrowth. In: Ecological Economics 70 (5), 873-880.

Klein, Dieter (2013): Das Morgen tanzt im Heute. Transformation im Kapitalismus und über ihn hinaus. Hamburg: VSA.

Jochimsen, Maren A.; Knobloch, Ulrike (Hrsg., 2006): Lebensweltökonomie in Zeiten ökonomischer Globalisierung. München.

Köhler, Bettina; Wissen, Markus (2010): Gesellschaftliche Naturverhältnisse. In: Lösch, Bettina; Thimmel, Andreas (Hg.): Kritische politische Bildung. Schwalbach, 217-227.

Latouche, Serge (2009): Farewell to Growth. Cambridge.

Layard, Richard (2009): Die glückliche Gesellschaft. Frankfurt/M. und New York.

Lötzer, Ulla; Reuter, Norbert (2012): Wachstumskritik. In: Brand, Ulrich et al. (Hg.): ABC der Alternativen. Hamburg: 322-3.

Mahnkopf, Birgit (2012): Kapitalismuskritik als Wachstumskritik. In: Dörre, Klaus; Sauer, Dieter; Wittke, Volker (Hg.): Kapitalismustheorie und Arbeit. Frankfurt/Main, 389-409

Martínez Alier, Joan; Pascual, Unai; Vivien, Franck-Dominique; Zaccai, Edwin (2010): Sustainable de-growth. Ecological Economics 69 (9), 1741-1747.

Meadows, D.H,; Meadows, D.L.; Randers, J.; Behrens, W.W., 1972. The Limits to Growth: A Report for the Club of Rome's Project on the Predicament of Mankind. New York.

MEW - Marx-Engels-Werke, Band 3 (1969): Die Deutsche Ideologie. Berlin.

-, Band 23 und 25 (1969): Das Kapital. Erster und Dritter Band. Berlin.

Miegel, Meinhard (2010): Exit. Wohlstand ohne Wachstum. Berlin.

Mitchell, Timothy (2011): Carbon Democracy: Political Power in the Age of Oil. London.

Muraca, Barbara (2012): Towards a fair degrowth-society: Justice and the right to a 'good life' beyond growth. In: Futures 44(6), 535-545.

- (2013): Décroissance: A Project for a Radical Transformation of Society. In: Environmental Values 22(2), 147-169.

Müller, Michael (2011): Aufklärung, Emanzipation, Fortschritt. Drucksache 17(26)31 der EnqueteKommission Wachstum, Wohlstand, Lebensqualität des Deutschen Bundestages.

NEF - New Economics Foundation (2010): The Great Transition. London.

OECD (2011): How's Life? Measuring well-being. Paris. 
Paech, Niko (2012): Befreiung vom Überfluss. München.

- (2005): Nachhaltiges Wirtschaften jenseits von Innovationsorientierung und Wachstum. Marburg.

Schmelzer, Mathias; Passadakis, Alexis (2011): Postwachstum. Krise, ökologische Grenzen und soziale Rechte. Hamburg.

Pennekamp, Johannes (2011): Wohlstand ohne Wachstum. Ein Literaturüberblick. Working Paper 1. Köln.

Reuter, Norbert (2012): Von der Wohlstands- zur reinen Wachstumsenquete? In: Gegenblende 15, Mai/Juni.

Rockström, J. et al. (2009): Planetary Boundaries. In: Ecology and Society 14(2).

Schneider, Francois; Kallis, Giorgos; Martinez Alier, Joan (2010): Crisis or Opportunity? Economic Degrowth for Social Equity and Ecological Sustainability. In: Journal of Cleaner Production 18(6), 511-518.

Spash, Clive (2012): New foundations for ecological economics. In: Ecological Economics 77, 36-47. SSFC - Stiglitz, Joseph; Sen, Amartya; Fitoussi, Jean Paul (2009): Report by the Commission on the Measurement of Economic Performance and Social Progress. (http://www.stiglitz-sen-fitoussi.fr)

Steurer, Reinhard (2010): Die Wachstumskontroverse als Endlosschleife. In: Wirtschaftspolitische Blätter 4, 423-435.

Svampa, Maristella (2012): Resource Extractivism and Alternatives: Latin American Perspectives on Development. In: Journal für Entwicklungspolitik (28)3, 43-73.

Thie, Hans (2013): Rotes Grün. Pioniere und Prinzipien einer ökologischen Gesellschaft. Hamburg.

UNEP (2011a): Decoupling Natural Resource Use and Environmental Impacts from Economic Growth. Report by the International Resource Panel. UNEP.

Welzer, Harald (2011): Mentale Infrastrukturen. Wie das Wachstum in die Welt und in die Seelen kam. Berlin: Heinrich-Böll-Stiftung.

- (2013): Selbst denken. Eine Anleitung zum Widerstand. Frankfurt/M.: S. Fischer.

Wichterich, Christa (2011): Kapitalismus mit Wärmedämmung. Feministische Kritik und Gegenentwürfe zur Green Economy. In: informationen für die frau, Oktober. Berlin: 5-7.

Wilkinson, Richard; Pickett, Kate (2010): Gleichheit ist Glück. Berlin.

Wissen, Markus (2011): Gesellschaftliche Naturverhältnisse in der Internationalisierung des Staates. Münster.

Zimmer, Matthias (2011): Fortschritt als bürgerliche Leitvorstellung. Drucksache 17(26)29 der Enquete-Kommission Wachstum, Wohlstand, Lebensqualität des Deutschen Bundestages. 